\title{
Phase diagrams of systems with two coupled order parameters
}

\author{
D. Nicolaides ${ }^{\dagger}$ and A.A. Lisyansky ${ }^{\ddagger}$ \\ ${ }^{\dagger}$ Natural Sciences and Mathematics, Bloomfield College, 467 \\ Franklin Street,Bloomfield, NJ 07003 \\ ${ }^{\ddagger}$ Department of Physics, Queens College of City University of \\ New York, Flushing, NY 11367
}

(February 24, 2018)

\begin{abstract}
Within the framework of an exactly solvable model, which takes into account the interaction of fluctuating modes with equal and opposite momenta, we consider phase diagrams in systems with coupled scalar order parameters. We show that, in agreement with the renormalization group theory, the fluctuation interaction can split the continuous disorder-order transition into two phase transitions of the first order. Moreover, the transition may occur into the anomalous, from the mean field theory point of view phase. The effect disappears when the fluctuation interaction is suppressed.
\end{abstract}

Typeset using REVTEX 


\section{INTRODUCTION}

Renormalization group ( $\mathrm{RG}$ ) theory made tremendous progress in understanding the physics of phase transitions of the second order (for review see, e.g., [1 [0] ). It allows one to calculate critical exponents with a very high degree of accuracy [6 9]. Moreover, it predicts a series of qualitative effects, such as the transformation of a continuous phase transition in some anisotropic systems into jump-like phase transitions due to the fluctuation interaction [2, 3,5]. Unfortunately, these transformations cannot be proven rigorously within the framework of RG. It is just a general belief that a flow of RG trajectories out of the region of stability of a Ginzburg-Landau-Wilson functional corresponds to a first order phase transition driven by fluctuations. There is a possibility that either such a treatment is incorrect or that such a flow away is a result of approximations, e.g., the $\epsilon$-expansion. Therefore, the treatment of these systems with the help of exactly solvable models, which at least partially take into account fluctuation effects, may clarify the situation. If an exactly solvable model yields results analogous to the ones of RG theory, it would be a serious argument in support of the reality of these qualitative effects.

In this paper we consider phase transitions in a system with two coupled scalar order parameters described by the Ginzburg-Landau-Wilson functional

$$
F\left(\phi_{1}, \phi_{2}\right)=\frac{1}{2} \int d^{3} x\left\{\sum_{i=1}^{2}\left[\tau_{i} \phi_{i}^{2}(\mathbf{x})+c_{i}\left(\nabla \phi_{i}(\mathbf{x})\right)^{2}+\frac{1}{4} g_{i} \phi_{i}^{4}(\mathbf{x})\right]+\frac{1}{2} w \phi_{1}^{2}(\mathbf{x}) \phi_{2}^{2}(\mathbf{x})\right\},
$$

where $\tau_{i}=\left(T-T_{c i}\right) / T_{c 1}$, and $T_{c i}$ is a trial critical temperature for the field $\phi_{i}(\mathbf{x})$. According to the mean field theory, lines $\tau_{1}=0$ and $\tau_{2}=0$ on the $\left(\tau_{1}, \tau_{2}\right)$ plane are lines of the second order phase transitions into phases $\phi_{1} \neq 0, \phi_{2}=0$ and $\phi_{2} \neq 0, \phi_{1}=0$, respectively [Fig. 1] [10]. However, the RG analysis shows that disorder-order phase transitions in the system described by the functional (1) can be of the first order [2, 1, 11, 12]. Our goal is to compare results of the RG theory with results of the exactly solvable model described below.

Our model uses a simplified physical picture of phase transitions. While preserving the symmetry of the system, it takes into account interaction of fluctuations with equal and opposite momenta only. This can be provided by splitting a $\delta$-function, $\delta\left(\mathbf{p}_{1}+\mathbf{p}_{2}+\mathbf{p}_{3}+\mathbf{p}_{4}\right)$, which is responsible for the momentum conservation, into a product of two $\delta$-functions, $\delta\left(\mathbf{p}_{1}+\mathbf{p}_{2}\right)\left(\mathbf{p}_{3}+\mathbf{p}_{4}\right)$. In coordinate space such a splitting corresponds to the following reduction of the interaction terms in the functional (11)

$$
\int d^{3} x \phi^{4}(\mathbf{x}) \rightarrow \frac{1}{V}\left[\int d^{3} x \phi^{2}(\mathbf{x})\right]^{2},
$$

where $V$ is the volume of the system. In its simplest version the model has the same critical asymptotics [13, 14] as the spherical model [15]. However, for more complicated systems it explicitly demonstrates major qualitative results that have been obtained within the RG theory, including fluctuation induced first order phase transitions [16, 17] and dimensional crossover for random systems [18].

\section{GENERAL RELATIONS}

In the functional (11) we reduce the interaction terms as follows, 


$$
\begin{aligned}
\int d^{3} x \phi_{i}^{4}(\mathbf{x}) & \rightarrow V a_{i}^{2}\left[\phi_{i}\right], \\
\int d^{3} x \phi_{1}^{2}(\mathbf{x}) \phi_{2}^{2}(\mathbf{x}) & \rightarrow V a_{1}\left[\phi_{1}\right] a_{2}\left[\phi_{2}\right],
\end{aligned}
$$

where

$$
a_{i}\left[\phi_{i}\right]=\frac{1}{V} \int d^{3} x \phi_{i}^{2}(\mathbf{x})
$$

After reduction (3) the exponent in the partition function becomes a quadratic form with respect to the functionals $a_{i}\left[\phi_{i}\right]$ :

$$
\begin{aligned}
Z & \propto \int D \phi_{1}(\mathbf{x}) D \phi_{2}(\mathbf{x}) \\
& \times \exp \left\{-\frac{V}{2}\left[\sum_{i=1}^{2}\left(\tau_{i} a_{i}\left[\phi_{i}\right]+\frac{1}{V} \int d^{3} x c_{i}\left(\nabla \phi_{i}(\mathbf{x})\right)^{2}+\frac{1}{4} g_{i} a_{i}^{2}\left[\phi_{i}\right]\right)+\frac{1}{2} w a_{1}\left[\phi_{1}\right] a_{2}\left[\phi_{2}\right]\right]\right\} .
\end{aligned}
$$

Using a transformation analogous to that of Hubbard-Stratanovich, we obtain:

$$
\begin{aligned}
Z & \propto \int D \phi_{1 \mathbf{q}} D \phi_{2 \mathbf{q}} \int_{-\infty}^{\infty} d x_{1} d x_{2} \int_{-i \infty}^{i \infty} d y_{1} d y_{2} \\
& \times \exp \left\{-\frac{V}{2} \sum_{i=1}^{2}\left[-i y_{i}\left(x_{i}-a_{i}\left[\phi_{i}\right]\right)+\tau_{i} x_{i}+\frac{1}{V} \sum_{\mathbf{q}} c_{i} q^{2}\left|\phi_{i \mathbf{q}}\right|^{2}+\frac{1}{4} g_{i} x_{i}^{2}\right]+\frac{1}{2} w x_{1} x_{2}\right\} .
\end{aligned}
$$

We may now perform integrations over all modes except $\phi_{i, \mathbf{q}=0}$, which may condense at the point of the phase transition, to obtain

$$
\begin{aligned}
Z & \propto \int_{-\infty}^{\infty} d \phi_{10} d \phi_{20} d x_{1} d x_{2} \int_{-\infty}^{\infty} d y_{1} d y_{2} \\
& \times \exp \left\{-\frac{V}{2} \sum_{i=1}^{2}\left[x_{i}\left(\tau_{i}-y_{i}\right)+\frac{1}{4} g_{i} x_{i}^{2}+\frac{1}{4} w x_{1} x_{2}+y_{i} \phi_{i 0}^{2}+\frac{1}{V} \sum_{\mathbf{q} \neq 0} \ln \left|c_{i} q^{2}+y_{i}\right|\right]\right\},
\end{aligned}
$$

where we define $\phi_{i 0}=\phi_{i \mathbf{q}=0} / \sqrt{V}$. The term $\sum_{\mathbf{q}} \ln \left|c_{i} q^{2}+y_{i}\right|$ in Eq. (6) diverges on the upper limit. Strictly speaking, this limit is finite and is equal to the cutoff momentum of the problem (which, e.g., in solids is a quantity of the order of the inverse lattice constant). However, critical asymptotics should not depend upon the cutoff momentum. Therefore, we renormalize sums over $\mathbf{q}$ making them finite and absorb divergencies into renormalizations of the trial critical temperatures. The renormalization can be done as follows,

$$
\sum_{\mathbf{q} \neq 0} R \ln \left(c_{i} q^{2}+y_{i}\right)=\sum_{\mathbf{q} \neq 0} \ln \left|c_{i} q^{2}+y_{i}\right|-\sum_{\mathbf{q} \neq 0} \ln \left(c_{i} q^{2}\right)-\sum_{\mathbf{q} \neq 0} \frac{y_{i}}{c_{i} q^{2}} .
$$

The sum on the left hand side of Eq. (7) is convergent even if we set the upper limit equal to infinity. The second term at the right side is an unimportant constant, and the third term causes renormalizations of the trial critical temperatures. As a result we have the partition function in the form, 


$$
Z \propto \int\left(\prod_{i} d \phi_{i 0} d x_{i} d y_{i}\right) \exp \left[-V F\left(x_{i}, y_{i}, \phi_{i 0}\right)\right],
$$

with function $F$ defined to be,

$$
F\left(x_{i}, y_{i}, \phi_{i 0}\right)=\frac{1}{2} \sum_{i=1}^{2}\left[x_{i}\left(t_{i}-y_{i}\right)+\frac{1}{4} g_{i} x_{i}^{2}+\frac{1}{4} w x_{1} x_{2}+y_{i} \phi_{i 0}^{2}+\Phi_{i}\left(y_{i}\right)\right],
$$

where

$$
\begin{aligned}
\Phi_{i}\left(y_{i}\right) & =\frac{1}{V} \sum_{\mathbf{q} \neq 0} R \ln \left(c_{i} q^{2}+y_{i}\right)=-\frac{4}{3} \kappa_{i} y_{i}^{3 / 2}, \\
\kappa_{i} & =\left(8 \pi c_{i}^{3 / 2}\right)^{-1},
\end{aligned}
$$

$t_{i}$ are renormalized critical temperatures,

$$
t_{i}=\tau_{i}+\frac{g_{i}}{2 V} \sum_{\mathbf{q} \neq 0} \frac{1}{c_{i} q^{2}}+\frac{w}{2 V} \sum_{\mathbf{q} \neq 0} \frac{1}{c_{j} q^{2}} \equiv \frac{T-T_{i}}{T_{1}}
$$

where $i=1,2$ when $j=2,1$.

Due to the fact that $V$ is a large multiplicative constant, one can use the steepest descent method to calculate the partition function exactly. The saddle points of the integral (8) are defined by the equations,

$$
\begin{aligned}
\frac{\partial F}{\partial x_{i}} & =\frac{1}{2}\left(t_{i}-y_{i}+\frac{g_{i} x_{i}}{2}+\frac{w x_{j \neq i}}{2}\right)=0, \\
\frac{\partial F}{\partial y_{i}} & =\frac{1}{2}\left(-x_{i}+\phi_{i 0}^{2}-f\left(y_{i}\right)\right)=0, \\
\frac{\partial F}{\partial \phi_{i 0}} & =y_{i} \phi_{i 0}=0,
\end{aligned}
$$

where

$$
f\left(y_{i}\right) \equiv-\frac{d \Phi_{i}}{d y_{i}}=2 \kappa_{i} y_{i}^{1 / 2}
$$

Solving this system of equations one can find the equilibrium values of $x_{i}, y_{i}$, and $\phi_{i 0}$. With these values Eq. (9) represents the equilibrium free energy per unit volume of the system.

Eliminating $x_{i}$ from Eqs. (10) we obtain a simplified system,

$$
\begin{aligned}
t_{i}-y_{i}+\frac{g_{i}}{2}\left[\phi_{i 0}^{2}-f\left(y_{i}\right)\right]+\frac{w}{2}\left[\phi_{j \neq i}^{2}-f\left(y_{j \neq i}\right)\right] & =0 \\
y_{i} \phi_{i 0} & =0
\end{aligned}
$$

with a free energy defined as

$$
\begin{aligned}
F\left(\phi_{i 0}\right)= & \frac{1}{2} \sum_{i=1}^{2}\left\{t_{i}\left[\phi_{i 0}^{2}-f\left(y_{i}\right)\right]+\frac{1}{4} g_{i}\left[\phi_{i 0}^{2}-f\left(y_{i}\right)\right]^{2}+\Phi_{i}\left(y_{i}\right)\right. \\
& \left.+\frac{1}{4} w\left[\phi_{10}^{2}-f\left(y_{1}\right)\right]\left[\phi_{20}^{2}-f\left(y_{2}\right)\right]+y_{i} f\left(y_{i}\right)\right\} .
\end{aligned}
$$




\section{PHASE DIAGRAMS}

According to the mean field theory there are two different types of phase diagrams for systems with coupled order parameters. They are the diagram with the bicritical point, where two lines of the second order phase transitions cross [Fig. 1a], and the tetracritical point diagram that has four crossed lines of phase transitions of the second order [Fig. 1b]. In the first case there are two ordered phases, $\phi_{10} \neq 0, \phi_{20}=0$ and $\phi_{10}=0, \phi_{20} \neq 0$, that are separated by a line of the first order phase transition. At the second case there is an additional ordered phase, $\phi_{10} \neq 0, \phi_{20} \neq 0$, which is separated from the other two phases by lines of the second order phase transitions. Below we reconstruct these diagrams in the framework of the exactly solvable model.

\section{A. Bicritical point phase diagram}

This diagram is the most interesting one since it can be substantially modified by the interaction of fluctuations. There are four different solutions of Eqs. (11) and (12). They correspond to the disordered phase, $\phi_{10}=0, \phi_{20}=0$, and three to the ordered phases: $\phi_{10} \neq 0, \phi_{20}=0, \phi_{10}=0, \phi_{20} \neq 0$ and $\phi_{10} \neq 0, \phi_{20} \neq 0$. In the case of the bicritical point phase diagram, when $\Delta=g_{1} g_{2}-w^{2}<0$, only ordered phases corresponding to the second and the third solutions may occur. Since they are symmetrical, let us consider, e.g., the phase $\phi_{10} \neq 0, \phi_{20}=0$.

This solution, according to Eq. (12), requires $y_{1}=0, y_{2} \neq 0$. In this case we have:

$$
y_{2 \pm}^{1 / 2}=-\frac{\kappa_{2} \Delta}{2 g_{1}} \pm \sqrt{\left(\frac{\kappa_{2} \Delta}{2 g_{1}}\right)^{2}+t_{2}-\frac{w t_{1}}{g_{1}}}
$$

with the following equilibrium values of the order parameter and the free energy

$$
\begin{aligned}
\phi_{10 \pm}^{2} & =\frac{2}{g_{1}}\left[-t_{1}-\frac{w \kappa_{2}^{2} \Delta}{2 g_{1}} \pm w \kappa_{2} \sqrt{\left(\frac{\kappa_{2} \Delta}{2 g_{1}}\right)^{2}+t_{2}-\frac{w t_{1}}{g_{1}}}\right] \\
F_{ \pm}\left(\phi_{10} \neq 0, \phi_{2}=0\right) & =-\frac{t_{1}^{2}}{g_{1}}+2 \kappa_{2}\left(\frac{w t_{1}}{g_{1}}-t_{2}\right) y_{2 \pm}^{1 / 2}+\frac{\Delta \kappa_{2}^{2}}{g_{1}} y_{2 \pm}+\frac{2}{3} \kappa_{2} y_{2 \pm}^{3 / 2} .
\end{aligned}
$$

When subscripts 1 and 2 are interchanged, $1 \leftrightarrow 2$, we obtain solutions for the phase $\phi_{10}=0$, $\phi_{20} \neq 0$. It can be shown that the free energy $F_{+}$is always lower than $F_{-}$. Therefore, only the solution $\phi_{10+} \neq 0, \phi_{20}=0, y_{1+}=0, y_{2+} \neq 0\left(\right.$ or $\left.\phi_{10}=0, \phi_{20+} \neq 0, y_{1+} \neq 0, y_{2+}=0\right)$ may realize.

According to the mean field theory the phase transition into an ordered phase is a continues one. However, as one can see from Eq. (16), the model predicts that this transition is of the first order if the following conditions are satisfied

$$
\begin{gathered}
w>g_{1}, \quad w>g_{2}, \\
T_{1}\left[\left(\frac{\kappa_{2} \Delta}{2 g_{1}}\right)^{2} \frac{w-g_{2}}{g_{2}}-1\right]>T_{2}\left[\left(\frac{\kappa_{1} \Delta}{2 g_{2}}\right)^{2} \frac{w-g_{1}}{g_{1}}-1\right],
\end{gathered}
$$




$$
4 g_{1}^{2}\left(T_{2}-T_{1}\right)+\kappa_{2}^{2}|\Delta|\left(w^{2}+g_{1} g_{2}-2 w g_{1}\right) T_{1}>0 .
$$

They are obtained by requiring that phase $\phi_{10+} \neq 0, \phi_{20}=0$ has a higher critical temperature than phase $\phi_{10}=0, \phi_{20+} \neq 0$, and that the jump of order parameter $\phi_{10+}$ is positive.

The transition occurs at the temperature defined by the equation

$$
\left(\frac{\kappa_{2} \Delta}{2 g_{1}}\right)^{2}+t_{2}-\frac{w t_{1}}{g_{1}}=0
$$

or

$$
T_{c}=\left\{\left[\left(\frac{\kappa_{2} \Delta}{2 g_{1}}\right)^{2}+\frac{w}{g_{1}}\right] T_{1}-T_{2}\right\}\left(\frac{w}{g_{1}}-1\right)^{-1} .
$$

The jump of the order parameter at the point of transition is equal

$$
\phi_{10}^{2}\left(T_{c}\right)=\frac{2}{g_{1}} \frac{T_{1}\left[1+\left(\frac{\kappa_{2} \Delta}{2 g_{1}}\right)^{2}\right]-T_{2}}{T_{1}\left(\frac{w}{g_{1}}-1\right)}+\frac{\kappa_{2} w|\Delta|}{g_{1}^{2}} .
$$

Such a transition occurs not necessarily into the phase with a higher trial critical temperature. Comparing free energies for the phases $\phi_{10} \neq 0, \phi_{20}=0$ and $\phi_{10}=0, \phi_{20} \neq 0$ one can show that for

$$
T<T_{c} \quad \text { and } \quad T_{1}<T_{2},
$$

and when conditions of inequalities (18) are true, the lower energy corresponds to the "anomalous", from the mean field theory point of view, phase $\phi_{10} \neq 0, \phi_{20}=0$. Only if $T_{2}<T_{1}$ the transition happens into the "normal" phase, $\phi_{10} \neq 0, \phi_{20}=0$.

The analogous result has been obtained in the RG theory [5, 12] where it was shown that in the system with competing order parameters, when $\Delta<0$ and trial critical temperatures, $T_{1}$ and $T_{2}$, are close to each other, the transition into the ordered phase is of the first order into the anomalous phase with the lower trial critical temperature. Such a phase is stabilized by the interaction of critical fluctuations, and when the temperature decreases further another phase transition into the "normal" phase must occur. Since the transition into the "normal" phase is the transition of the order-order type it must also be of the first kind. Hence, the fluctuation interaction splits the continuous phase transition, predicted by the mean field theory, into two transitions of the first order. The effect must disappear when fluctuations are suppressed. The model provides a simple way of regulating the strength of fluctuations. Fluctuations are suppressed in systems with long-range interactions. The parameters $c_{i}^{1 / 2}$ represent the radii of interactions in the original system in which critical behavior is described by Ginzburg-Landau functional (1). The Ginzburg number, Gi, that defines the width of the domain where fluctuation are essential, is proportional to $c_{i}^{-3}$ or $\kappa_{i}^{2}$. So, setting $\kappa_{i} \rightarrow 0$ we suppress fluctuations. In this case the order parameter (16) becomes proportional to $\sqrt{-t_{1}}$ and the transition becomes of the second order. It is interesting to note that the effect of inducing the first order transition into the phase $\phi_{10} \neq 0, \phi_{20}=0$ is controlled only by the fluctuations of the order parameter $\phi_{20}$ (and, of course by the coupling, $w$, between modes). Using the model, the phase diagram corresponding to the first order phase transition into the anomalous phase is schematically shown in Fig. 2. 


\section{B. Tetracritical point phase diagram}

In the mean field theory the case of $\Delta>0$ corresponds to the phase diagram with the tetracritical point shown in Fig. 1b. According to the model, the phase $\phi_{10} \neq 0, \phi_{20}=0$ corresponds to the solution of Eqs. (11) and (12). The result is $\phi_{10+}$ defined by Eq. (16), which independently of the sign of $w$, always has a solution and the transition is of the second order. The critical temperature in this case is defined by

$$
T_{c}=T_{1}+\frac{w \kappa_{2}^{2}\left(w-g_{2}\right) T_{1}}{2}+\frac{w \kappa_{2} T_{1}}{2} \sqrt{\kappa_{2}^{2}\left(w-g_{2}\right)^{2}+\frac{4\left(T_{1}-T_{2}\right)}{T_{1}}} .
$$

The RG theory, however, allows for a fluctuation induced first order phase transition when $\Delta>0$ and $w<0$. This discrepancy can be attributed to that the model takes into account fluctuations in a limited manner.

The transition between ordered phases is described by Eqs. (11) and (12) with $y_{1}=$ $y_{2}=0$ :

$$
\begin{aligned}
& t_{1}+\frac{g_{1}}{2} \phi_{1}^{2}+\frac{w}{2} \phi_{2}^{2}=0, \\
& t_{2}+\frac{g_{2}}{2} \phi_{2}^{2}+\frac{w}{2} \phi_{1}^{2}=0 .
\end{aligned}
$$

These equations have ordinary mean field theory solutions

$$
\begin{aligned}
& \phi_{10}^{2}=2\left(w t_{2}-g_{2} t_{1}\right) / \Delta, \\
& \phi_{20}^{2}=2\left(w t_{1}-g_{1} t_{2}\right) / \Delta,
\end{aligned}
$$

which exists when

$$
\Delta>0, \quad w t_{2}-g_{2} t_{1}>0, \quad w t_{1}-g_{1} t_{2}>0 .
$$

According to Eqs. (24) in agreement with the mean field theory transitions between ordered phases for positive $\Delta$ are of the second order. Besides the renormalization of critical temperatures, according to the model, fluctuations do not change the tetracritical point phase diagram. 


\section{REFERENCES}

[1] S.-k. Ma, Modern Theory of Critical Phenomena (Benjamin, NY, 1976).

[2] A. Aharony, in Phase Transitions and Critical Phenomena (Academic Press, NY, 1976).

[3] D. Amit, Field Theory, the Renormalization Group, and Critical Phenomena (World Scientific, Singapore, 1984).

[4] G.A. Baker, Jr., Quantitative Theory of Critical Phenomena (Academic Press, New York, 1990).

[5] Yu.M. Ivanchenko, A.A. Lisyansky and A.E. Filippov, Physics of Critical Fluctuations (Springer, New York, 1995).

[6] G.A. Baker, Jr., B. G. Nickel, and D. I. Meiron, Phys. Rev. B 17, 1365 (1978).

[7] S.G. Gorishny, S.A. Larin, and F.V. Tkachov, Phys. Lett. A 101, 120 (1984).

[8] C. Bervillier and C. Godreche, Phys. Rev. B 21, 5427 (1980).

[9] J.C. Le Guillon, and J. Zinn-Justin, J. Phys. (Paris) 48, 19 (1987).

[10] K.-S. Liu and M.E. Fisher, J. Low Temp. Phys. 10, 655 (1972).

[11] J.M. Kosterlitz, D.R. Nelson, and M.E. Fisher, Phys. Rev. B 13, 412 (1976).

[12] Yu.M. Ivanchenko, A.A. Lisyansky, and A.E. Filippov, Sov. Phys. - JETP 60, 582 (1984).

[13] T. Schneider, E. Stoll, and H. Beck, Physica A 34, 436 (1975).

[14] Yu.M. Ivanchenko, A.A. Lisyansky and A.E. Filippov, Theor. \& Math. Phys. 67, 413 (1986).

[15] T.H. Berlin and M. Kac, Phys. Rev. 86, 821 (1952).

[16] Yu.M. Ivanchenko, A.A. Lisyansky and A.E. Filippov, Phys. Lett. A 119, 55 (1986).

[17] A.A. Lisyansky and D. Nicolaides, Phys. Lett. A 173, 179 (1993).

[18] D. Nicolaides and A.A. Lisyansky, Phys. Rev. B 53, 8168 (1996). 


\section{FIGURE CAPTIONS}

Fig. 1. Phase diagrams with bicritical (a) and tetracritical (b) points in the mean field theory. For these and the next figure dashed lines correspond to continuous phase transitions, the solid line corresponds to a phase transition of the first order between two ordered phases.

Fig. 2. Bicritical point phase diagram modified due to fluctuations. Near the point where lines of the order-disorder transitions cross all phase transitions become of the first order. With decreasing temperature the state of the system moves along the dotted line. When this line crosses the first solid line, as shown, the disorder-order transition is of the first order into the "anomalous" phase instead of the continuous phase transition described by the mean field theory. When the dotted line crosses the second solid line the first order transition into the "normal" phase occurs. 


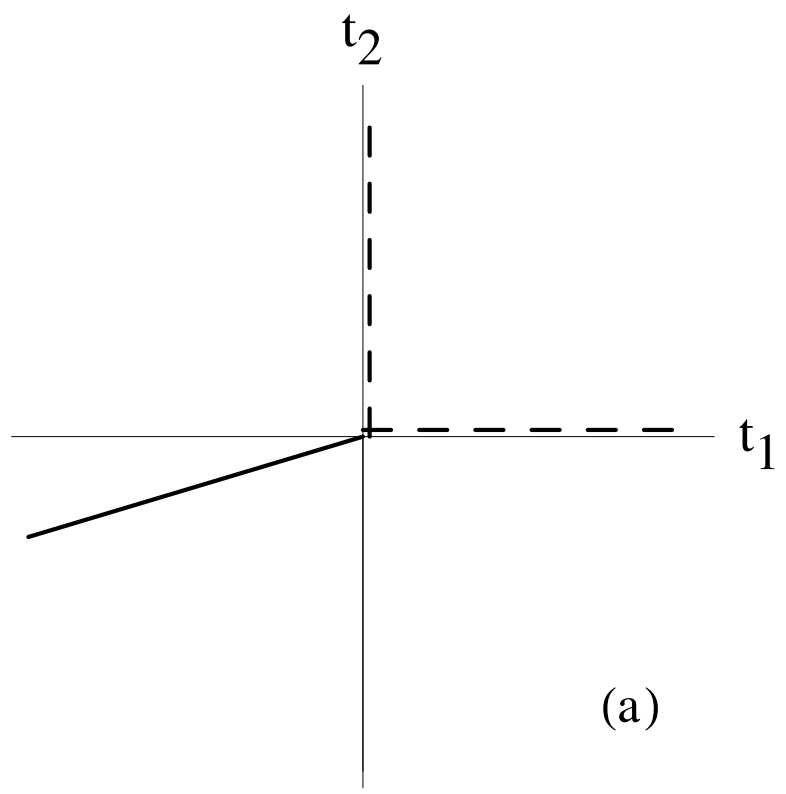




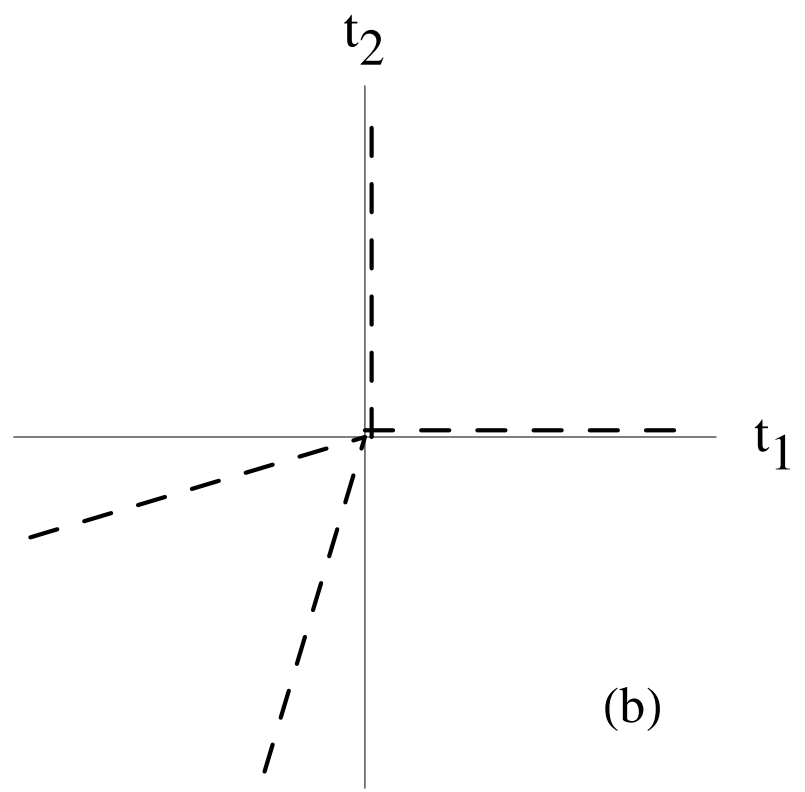




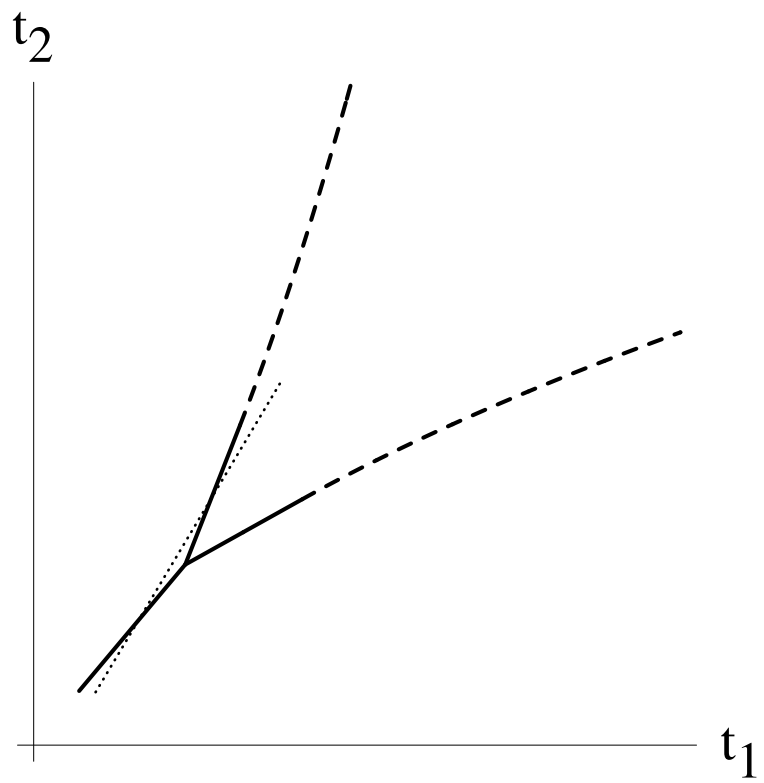

